\title{
Experience with Elizabethkingia meningoseptica Infection in Adult Patients at a Tertiary Hospital
}

\author{
Hyun Don Joo, M.D. ' , Sun Young Ann, M.D. ${ }^{1}$, Sung Hyeok Ryou, M.D. ${ }^{1}$, \\ Youn Seup Kim, M.D., Ph.D. ${ }^{1}$, Jong Wan Kim, M.D., Ph.D. ${ }^{2}$, and Doh Hyung Kim, M.D., Ph.D \\ ${ }^{1}$ Department of Internal Medicine, ${ }^{2}$ Department of Laboratory Medicine, Dankook University Hospital, Dankook University College of Medicine, Cheonan, Korea
}

Background: Few reports have documented the clinical characteristics and treatment outcomes of adult patients with Elizabethkingia meningoseptica infection.

Methods: Medical records of patients over 18 years of age and suspected of having an E. meningoseptica infection from March 1, 2006 to February 28, 2013 were reviewed retrospectively. Their clinical characteristics, antimicrobial susceptibility results, and treatment outcomes were analyzed.

Results: E. meningoseptica was isolated from 30 patients. Median age was 68.5 years, and infections were more frequent in males (17, $56.7 \%)$. The most common isolation source was sputum (23,76.7\%), and pneumonia was the most common condition ( $21,70 \%)$ after excluding two cases of colonization. This bacterium was most susceptible to minocycline $(27,90 \%)$ and fluoroquinolones, including levofloxacin $(20,66.7 \%)$ and ciprofloxacin $(18,60 \%)$. The mortality rate due directly to E. meningoseptica infection was $20 \%(6 / 30)$, and uncontrolled pneumonia was the only cause of death. After isolating E. meningoseptica, the numbers of patients with pneumonia $(9 / 9$, $100 \%$ vs. $12 / 21,57.1 \%)$, history of hemodialysis $(5 / 9,55.6 \%$ vs. $3 / 21,14.3 \%)$, tracheostomy $(8 / 9,88.9$ vs. $10 / 21,47.6 \%)$, and median Charlson comorbidity index score (6 [range, 3-9] vs. 4 [range, 0-9]) were significantly higher in non-survivors than those in survivors ( $p$ $<0.05$, for each). However, only $12(40 \%)$ patients received appropriate antibiotics.

Conclusions: $E$. meningoseptica infection most commonly presented as pneumonia in adults with severe underlying diseases. Despite the high mortality rate, the rate of appropriate antibiotic use was notably low.

Key Words: Chryseobacterium meningosepticum; Elizabethkingia meningoseptica; Flavobacterium meningosepticum; nosocomial infection; pneumonia.

\section{Introduction}

The increasing emergence of antibiotic-resistant nosocomial infections is a worldwide concern. Elizabethkingia meningoseptica (previously known as Flavobacterium meningosepticum or Cryseobacterium meningosepticum, Center for Disease Control and Prevention group II-a) is a non-fastidious oxidase-positive, non-glucose fermenting, Gram-negative aerobic rod bacterium that is widely distributed in soil, plants, and water

Received on February 23, 2015 Revised on May 26, 2015 Accepted on June 7, 2015

Correspondence to: Doh Hyung Kim, Department of Internal Medicine, College of Medicine, Dankook University, 119 Dandae-ro, Dongnam-gu, Cheonan 31116, Korea

Tel: +82-41-550-3870, Fax: +82-41-556-3256

E-mail: kimdh@dankook.ac.kr

*No potential conflict of interest relevant to this article was reported. but is not normally found in human microflora.[1] It is the most pathogenic Cryseobacterium sp. capable of infecting humans and infects the blood, cerebrospinal fluid, skin, soft tissues, respiratory system, and other sites.[2,3] In addition, it reportedly exhibits inherent resistance to several classes of important antibiotics, such as beta-lactams, aminoglycosides, carbapenems, clindamycin, tetracyclines, chloramphenicol, 
erythromycin, and teicoplanin, which are the most common antibiotics used to treat Gram-negative nosocomial infections.[3] However, choosing an effective antibiotic against E. meningoseptica is clinically quite difficult because no interpretive minimum inhibitory concentration (MIC) breakpoints of antibiotics against this organism have been reported and reports of antimicrobial responses and treatment outcomes are rare.[3]

Although most reports of E. meningoseptica infection in humans have been presented as meningitis outbreaks in premature newborns and infants,[4-6] several reports have emphasized its importance as a nosocomial infection in adult patients with severe underlying disease.[7-11] However, studies evaluating the clinical significance and treatment outcomes in adult patients with E. meningoseptica infection are rare.

In this study, we evaluated the clinical characteristics and results of antimicrobial susceptibility testing in adult patients with E. meningoseptica infection and analyzed their treatment outcomes.

\section{Materials and Methods}

\section{1) Data collection}

This study was conducted retrospectively in patients admitted to Dankook University Hospital, a tertiary care and teaching hospital in South Korea, from March 1, 2006 to February 28, 2013. The study subjects were selected based on bacterial culture reports after excluding patients $<18$ years of age, and the demographic and clinical features were obtained from their medical records, including age, sex, main cause for admission, comorbidities, isolation sources, infection types, clinical conditions predisposing the patient to hospital infection, antimicrobial susceptibility test results, and treatment outcome. Intermediate strains were classified into resistant strains to collect the antimicrobial susceptibility results.

\section{2) Microbiology}

The Clinical and Laboratory Standards Institute did not establish definite interpretative MIC breakpoints for $E$. meningoseptica until 2013; thus, most previous reports presented susceptibility test results using MIC breakpoint results for Pseudomonas aeruginosa or non-Enterobacteriaceae sp.[12] In this study, The VITEK2 system (bioMerieux Vitek; bioMerieux, Marcy I'Etoile, France) with the VITEK2 GN and antimicrobial susceptibility test cards were used to identify bacteria and confirm antimicrobial susceptibility. Antimicrobial susceptibilities were defined for amikacin, aztreonam, cefepime, cefotaxime, ceftazidime, ciprofloxacin, colistin, gentamicin, imipenem, levofloxacin, meropenem, minocycline, piperacillin, piperacillin/tazobactam, and trimethoprim/sulfamethoxazole using MIC standards $(\mu \mathrm{g} / \mathrm{mL})$ for other non-Enterobacteriaceae, including Pseudomonas sp. (except $P$. aeruginosa) and other nonfastidious, glucosenonfermenting, Gram-negative bacilli, Acinetobacter sp., Burkhoderia cepacia, B. mallei, B. pseudomallei, and Stenotrophomonas maltophilia.

\section{3) Definitions}

"E. meningoseptica infection" was defined when the attending clinician decided to perform a bacterial culture and to started or changed the current antibiotic in patients with either a newly developed fever $\geq 38.3^{\circ} \mathrm{C}$ or leukocytosis $>10,000 / \mathrm{mm}^{3}$. "Colonization" was defined when patients did not meet the definition of "E. meningoseptica infection" described above and were stable without any symptoms or signs of infection after 1 week. "Pneumonia" was defined when patients met the definition of "E. meningoseptica infection" and showed both new infiltration on chest radiographs and documented changes in sputum color or quantity on medical records. "Prolonged hospitalization" was defined as hospitalization $>28$ days prior to isolating $E$. meningoseptica. "Prior intensive care unit (ICU) stay" and "mechanical ventilation" were defined when patients stayed in the ICU or were mechanically ventilated for $>48$ hours prior to isolating E. meningoseptica. "Recent surgery" was defined when a surgical procedure excluding tracheostomy was performed during the current hospitalization prior to isolating E. meningoseptica. "Appropriate antibiotic use" was defined when at least one susceptible intravenous antimicrobial agent was used within 48 hours after obtaining the culture report. "E. meningoseptica-related mortality" was defined when patients died within 2 weeks after $E$. meningoseptica was isolated, and no other explainable cause of death was found. 


\section{4) Statistical analysis}

The statistical analysis was performed using Fisher's exact test for categorical variables and the Mann-Whitney $U$ test for continuous variables. SPSS ver. 13.0 software (SPSS Inc., Chicago, IL, USA) was used for all analyses. A p $<0.05$ indicated statistical significance.

\section{Results}

Thirty patients were included in this study, and their demographic and clinical characteristics are summarized in Table 1. Median age was 68.5 years (range, 19-90 years), and males made up the majority of patients $(17,56.7 \%)$. The most common cause of the current admission was pneumonia $(7,23.3 \%)$, followed by intracranial hemorrhage $(5,16.7 \%)$, and malignancy $(5,16.7 \%)$. Twenty-one $(70 \%)$ patients had underlying comorbidities, including cardiovascular disease $(14 / 21,66.7 \%)$, malignancy $(6 / 21,28.6 \%)$, diabetes $(6 / 21,28.6 \%)$, chronic renal disease $(4 / 21,19.0 \%)$, steroid use (4/21, 19.0\%), and chronic lung disease (3/21, 14.3\%). Their median Charlson comorbidity index score was 5 (range, $0-9$ ). The most common isolation source was sputum $(23,76.7 \%)$, and pneumonia was the most common infection type (21, 70\%) after excluding two cases of colonization.

Prolonged hospitalization $>28$ days prior to isolating $E$. meningoseptica $(26,86.7 \%)$, prior ICU stay $(23,76.7 \%)$, and mechanical ventilation $(23,76.7 \%)$ were observed frequently in the study population among the variables used to evaluate which clinical conditions predisposed patients to developing the infection while in the hospital. The median duration and number of antibiotics used before isolating $E$. meningoseptica was 33 days (range, 1-216 days) and four (range, 1-11), respectively.

The in vitro antimicrobial susceptibility test results are presented in Table 2. E. meningoseptica was most often susceptible to minocycline $(27,90 \%)$ and fluoroquinolones, including levofloxacin $(20,66.7 \%)$ and ciprofloxacin (18, $60 \%$ ). However, most antibiotics that target Gram-negative bacteria were not very effective against $E$. meningoseptica.

Treatment outcomes are presented in Table 3. The allcause mortality rate after isolating E. meningoseptica was $30 \%(9 / 30)$, and the mortality rate assumed to be directly
Table 1. Demographic characteristics of the study population

\begin{tabular}{|c|c|}
\hline Characteristics & $\begin{array}{l}\text { Number (\%) } \\
\quad(\mathrm{N}=30)\end{array}$ \\
\hline Median age, years (range) & $68.5(19-90)$ \\
\hline Male & $17(56.7)$ \\
\hline \multicolumn{2}{|l|}{ Main causes of current admission } \\
\hline Pneumonia & $7(23.3)$ \\
\hline Intracranial hemorrhage & $5(16.7)$ \\
\hline Malignancy & $5(16.7)$ \\
\hline Bone and joint infection & $3(10.0)$ \\
\hline Cardiac diseases & $2(6.7)$ \\
\hline Renal diseases & $2(6.7)$ \\
\hline Others & $6(20.0)$ \\
\hline \multicolumn{2}{|l|}{ Comorbidities (may be multiple) $(\mathrm{N}=21$ ) } \\
\hline Cardiovascular disease & $14(66.7)$ \\
\hline Diabetes & $6(28.6)$ \\
\hline Malignancy & $6(28.6)$ \\
\hline Chronic renal disease & $4(19.0)$ \\
\hline Steroid use & $4(19.0)$ \\
\hline Chronic lung disease & $3(14.3)$ \\
\hline Adrenal insufficiency & $2(9.5)$ \\
\hline Cerebrovascular disease & $1(4.8)$ \\
\hline Charlson comorbidity index, median (range) & $5(0-9)$ \\
\hline \multicolumn{2}{|l|}{ Sources of isolation } \\
\hline Sputum & $23(76.7)$ \\
\hline Catheter & $5(16.7)$ \\
\hline Blood & $1(3.3)$ \\
\hline Urine & $1(3.3)$ \\
\hline \multicolumn{2}{|l|}{ Types of infection } \\
\hline Pneumonia & $21(70.0)$ \\
\hline Catheter related infection & $5(16.7)$ \\
\hline Urinary tract infection & $1(3.3)$ \\
\hline Bacteremia of undefined source & $1(3.3)$ \\
\hline Colonization & $2(6.7)$ \\
\hline \multicolumn{2}{|l|}{ Clinical conditions predisposing hospital infection } \\
\hline Prolonged hospitalization (> 28 days) & $26(86.7)$ \\
\hline Total duration (days) of previous antibiotic use, median (range) & $33(1-216)$ \\
\hline Number of previous antibiotics, median (range) & $4(1-11)$ \\
\hline Prior intensive care unit stay & $23(76.7)$ \\
\hline Mechanical ventilation & $23(76.7)$ \\
\hline Hemodialysis & $8(26.7)$ \\
\hline Tracheostomy & $18(60.0)$ \\
\hline Recent surgery & $14(46.7)$ \\
\hline
\end{tabular}

Data are presented as the median (range) or number (\%).

${ }^{*}$ Others include tuberculous pleurisy, peritonitis, soft tissue infection, encephalitis, spinal cord injury, and multiple fractures. 
Table 2. In vitro activities of the tested antimicrobial agents against Elizabethkingia meningoseptica isolates

\begin{tabular}{|c|c|c|c|c|}
\hline \multirow{2}{*}{ Antimicrobial agent } & \multicolumn{3}{|c|}{ MIC interpretive criteria $(\mu \mathrm{g} / \mathrm{mL})$} & \multirow{2}{*}{$\begin{array}{l}\text { No. (\%) of isolates susceptibl } \\
\qquad(\mathrm{N}=30)\end{array}$} \\
\hline & $\mathrm{S}$ & I & $\mathrm{R}$ & \\
\hline Amikacin & $\leq 16$ & 32 & $\geq 64$ & $1(3.3)$ \\
\hline Aztreonam & $\leq 8$ & 16 & $\geq 32$ & $0(0.0)$ \\
\hline Cefepime & $\leq 8$ & 16 & $\geq 32$ & $1(3.3)$ \\
\hline Cefotaxime & $\leq 8$ & $16-32$ & $\geq 32$ & $0(0.0)$ \\
\hline Ceftazidime & $\leq 8$ & 16 & $\geq 32$ & $1(3.3)$ \\
\hline Ciprofloxacin & $\leq 1$ & 2 & $\geq 4$ & $18(60.0)$ \\
\hline Colistin & $\leq 2$ & 4 & $\geq 8$ & $0(0.0)$ \\
\hline Gentamicin & $\leq 4$ & 8 & $\geq 16$ & $3(10.0)$ \\
\hline Imipenem & $\leq 4$ & 8 & $\geq 16$ & $0(0.0)$ \\
\hline Levofloxacin & $\leq 2$ & 4 & $\geq 8$ & $20(66.7)$ \\
\hline Meropenem & $\leq 4$ & 8 & $\geq 16$ & $1(3.3)$ \\
\hline Minocycline & $\leq 4$ & 8 & $\geq 16$ & $27(90.0)$ \\
\hline Piperacillin & $\leq 16$ & $32-64$ & $\geq 128$ & $1(3.3)$ \\
\hline Piperacillin/tazobactam & $\leq 16 / 4$ & $32 / 4-64 / 4$ & $\geq 32$ & $6(20.0)$ \\
\hline Trimethoprim/sulfamethoxazole & $\leq 2 / 38$ & & $\geq 4 / 76$ & $9(30.0)$ \\
\hline
\end{tabular}

Data are presented as number (\%).

MIC: minimal inhibitory concentration; S: susceptible; I: intermediate; R: resistant.

Table 3. Treatment outcomes and appropriate antibiotic use

\begin{tabular}{lc}
\hline Treatment outcomes & $\begin{array}{c}\text { Number (\%) } \\
(\mathrm{N}=30)\end{array}$ \\
\hline The all-cause mortality after isolation of E. meningoseptica & $9(30.0)$ \\
\hline E. meningoseptica -related mortality & $6(20.0)$ \\
Hypoxic brain death & $1(3.3)$ \\
Arrhythmia (ventricular tachycardia) & $1(3.3)$ \\
Massive hemoptysis & $1(3.3)$ \\
Appropriate antibiotic use based on culture reports & $12(40.0)$ \\
\hline $\begin{array}{l}\text { Data are presented as number (\%). } \\
{ }^{*} \text { All causes of death were defined as Elizabethkingia meningoseptica pneumonia. }\end{array}$
\end{tabular}

affected by E. meningoseptica infection was $20 \%(6 / 30)$. Uncontrolled pneumonia was defined as the only cause of E. meningoseptica-related mortality but three patients died of hypoxic brain injury, arrhythmia, and massive hemoptysis. Despite the high mortality, the appropriate antibiotics based on culture results were only provided to $12(40.0 \%)$ patients.

Differences in the clinical characteristics between survivors and non-survivors after isolating E. meningoseptica are shown in Table 4. The presence of Elizabethkingia pneumonia $(9 / 9,100 \%$ vs. $12 / 21,57.1 \%)$, median Charlson comorbidity index score (6 [range, 3-9] vs. 4 [range, 0-9])), history of hemodialysis $(5 / 9,55.6 \%$ vs. $3 / 21,14.3 \%)$, and tracheostomy $(8 / 9,88.9 \%$ vs. $10 / 21,47.6 \%)$ were significantly higher in non-survivors than those in survivors ( $\mathrm{p}<$ 0.05 , for each) based on a univariate analysis. E. meningoseptica infection presented as pneumonia in all non-survivors, and appropriate antibiotics based on antimicrobial susceptibility test results were not provided for any of the six $E$. meningoseptica-related deaths. Despite a lack of statistical significance, prolonged hospitalization, prior ICU stay, and the use of mechanical ventilation were more frequently observed in non-survivors than those in survivors.

\section{Discussion}

The role of E. meningoseptica in nosocomial infections, particularly among immunocompromised patients with severe underlying disease, has been raised continuously in previous reports, even though these infections are considered uncommon in adults.[7-11,13] However, information on the clinical significance and treatment outcomes in adults with E. meningoseptica nosocomial infection has 
Table 4. Comparison of survivors and non-survivors after isolating Elizabethkingia meningoseptica

\begin{tabular}{|c|c|c|c|}
\hline Clinical characteristics & $\begin{array}{c}\text { Survivors } \\
(\mathrm{N}=21)\end{array}$ & $\begin{array}{l}\text { Non-survivors } \\
\quad(\mathrm{N}=9)\end{array}$ & $\mathrm{p}$-value \\
\hline Median age, years (range) & $67.0(19-90)$ & $70.0(40-81)$ & 0.892 \\
\hline Male & $12(57.1)$ & $5(55.6)$ & 1.000 \\
\hline \multicolumn{4}{|l|}{ The most common site of isolation } \\
\hline Sputum & $14(66.7)$ & $9(100.0)$ & 0.071 \\
\hline Others & $7(33.3)$ & $0(0.0)$ & \\
\hline \multicolumn{4}{|l|}{ The most common type of infection } \\
\hline Pneumonia & $12(57.1)$ & $9(100.0)$ & 0.029 \\
\hline Others & $9(42.9)$ & $0(0.0)$ & \\
\hline Appropriate antibiotic use based on culture results ${ }^{\dagger}$ & $9(42.9)$ & $3(33.3)$ & 0.704 \\
\hline \multicolumn{4}{|c|}{ Clinical conditions predisposing hospital infection during current admission } \\
\hline Median Charlson comorbidity index (range) & $4(0-9)$ & $6(3-9)$ & 0.033 \\
\hline Prolonged hospitalization (> 28 days) & $17(81.0)$ & $9(100.0)$ & 0.287 \\
\hline Total duration (days) of previous antibiotic use, median (range) & $32(2-216)$ & $44(1-107)$ & 0.751 \\
\hline Number of previous antibiotics, median (range) & $4(2-11)$ & $4(1-11)$ & 0.766 \\
\hline Prior intensive care unit stay & $14(66.7)$ & $9(100.0)$ & 0.071 \\
\hline Mechanical ventilation & $14(66.7)$ & $9(100.0)$ & 0.071 \\
\hline Hemodialysis & $3(14.3)$ & $5(55.6)$ & 0.032 \\
\hline Tracheostomy & $10(47.6)$ & 8 (88.9) & 0.049 \\
\hline Recent surgery & $11(52.4)$ & $3(33.3)$ & 0.440 \\
\hline
\end{tabular}

Data are presented as the median (range) or number (\%).

*Among nine patients with pneumonia, E. meningoseptica pneumonia was the direct cause of death in six, and the others died due to hypoxic brain injury, arrhythmia, and massive hemoptysis.

${ }^{\dagger}$ Appropriate antibiotics were not provided for any of the six E. meningoseptica-related deaths.

been scarce. Only a few case studies of E. meningoseptica infection have bee reported in Korea, including patients with keratitis, peritonitis, ventriculitis, and meningitis.[1420] In this study, we evaluated the clinical characteristics, antimicrobial susceptibility, and treatment outcomes in adult patients suspected to have an E. meningoseptica infection at a tertiary hospital. Nosocomial infection by E. meningoseptica in adult patients was not as uncommon as we expected, particular in patients with severe underlying diseases, a history of prolonged hospitalization, an ICU stay, and who used mechanical ventilation. Pneumonia was the most common infection caused by E. meningoseptica, and the mortality rate was quite high. However, despite the high mortality rate, the rate of appropriate antibiotic use was notably low.

Increasing incidence of antibiotic resistance by nosocomial infections is a global concern due to the increased use of broad-spectrum antibiotics. E. meningoseptica is intrinsically resistant to most $\beta$-lactams commonly used to treat nosocomial infections. Chromosomally linked metallo- $\beta$ lactamases and multiple heterogeneous carbapenem-hydrolyzing enzymes have been identified as key antibiotic resistance factors in E. meningoseptica, [3,21,22] and one report suggested that carbapenem resistance by E. meningoseptica is mediated by metallo- $\beta$-lactamase BlaB.[23] Because of its resistance to carbapenem, increasing use of this antibiotic for severe nosocomial infections caused by extendedspectrum $\beta$-lactamase-producing Gram-negative bacteria, such as Pseudomonas and Acinetobacter spp., might contribute to the emergence of E. meningoseptica infection, particularly in the ICU. Research from Taiwan reported an increased incidence of bacteremia caused by E. meningoseptica that developed mostly in adult hospitalized patients between 1999 and 2006, with an incidence rate of 7.5-35.6 per 100,000 admissions. [7] Considering the difficulty isolating this bacterium from clinical specimens and the relatively low accuracy $(74.8 \%)$ of culture, $[3,24]$ healthcare providers 
need to monitor the probability for an increased incidence of nosocomial infections caused by E. meningoseptica in patients at risk.

E. meningoseptica is a waterborne saprophytic bacterium that is widely distributed in nature and in hospitals. Although the mode of transmission is not well known, various reports have suggested a possible association between contaminated water, mechanical ventilation respiratory equipment, and indwelling devices and hospital outbreaks.[1,25] In support of these previous suggestions, E. meningoseptica was most frequently isolated from respiratory specimens $(23 / 30,76.7 \%)$ and usually manifested as pneumonia (21/30, $70 \%$ ) in our study population. There was a possibility of overestimating the incidence of pneumonia in our study because of the limitations of a retrospective study design. A more accurate method, such as a clinical pulmonary infection score, to define the presence of pneumonia was not applied in most patients. However, E. meningosepticarelated mortality was evaluated completely after reviewing all nurse and doctor records as well as laboratory and radiological test results, and no other explainable causes of death were found, except E. meningoseptica pneumonia. The $20 \%$ (6/30) rate of E. meningoseptica-related mortality in our study population was similar to the $23 \%$ rate observed in the majority of adults in a previous report.[7] Based on these findings, pneumonia may be the most common type of $E$. meningoseptica infection in adults and isolating $E$. meningoseptica from a respiratory specimen in a patients suspected to have pneumonia should not be considered contamination or colonization until a more probable causative organism is found.

Prolonged hospitalization $>28$ days $(86.7 \%)$, history of ICU stay (76.7\%), and mechanical ventilation (76.7\%) were frequently observed when we evaluated the clinical characteristics of our study population. In addition, the median Charlson comorbidity index score, prolonged hospitalization, total duration of previous antibiotic use, prior ICU stay, mechanical ventilation, hemodialysis, and a tracheostomy were observed at higher rates in non-survivors than those of survivors, despite the lack of statistical significance in some variables. These findings suggest that severe underlying illnesses and comorbidities that predispose patients to prolonged hospitalization could be risk factors for E. meningoseptica infection. However, the possibility that the high rate of all-cause mortality might be more affected by a patient's condition rather than the E. meningoseptica infection itself, indicating that the infection is just a sign of overall poor health, cannot be excluded completely.

No consensus has been reached about the drug of choice for treating patients with an E. meningoseptica infection because of the lack of interpretive MIC breakpoints and the rarity of studies that have evaluated the clinical response of antibiotics against the organism. Minocycline, ciprofloxacin, trimethoprim/sulfamethoxazole, rifampin, and new respiratory fluoroquinolones, such as levofloxacin, gatifloxacin, and piperacillin/tazobactam, have been suggested for treating E. meningoseptica based on in vitro susceptibility results.[1,3,8,10,18] Markedly discrepant results between the disc diffusion and broth microdilution methods for vancomycin using staphylococcal breakpoints has also been reported even though E. meningoseptica has been reported to be susceptible to antibiotics effective against Grampositive cocci.[26] These finding suggest unreliability of the disc diffusion method for determining E. meningoseptica antimicrobial susceptibility. As a consequence, vancomycin and other antibiotics against Gram positive cocci, including teicoplanin, linezolid, and quinupristin-dalfopristin are not recommended to treat this organism, except rifampin. $[3,26]$ A few reports have presented cases of E. meningoseptica infection that only responded to a combined antibiotic treatment, including piperacillin/tazobactam plus rifampin, vancomycin plus rifampin, or fluoroquinolone combined with vancomycin plus rifampin.[5,27-30] Thus, the role of vancomycin as a combination agent with other antibiotics has not been fully determined.

According to the SENTRY Antimicrobial Surveillance Program (1997-2001), which was a world-wide study about the susceptibility and resistance patterns of bacterial and fungal pathogens, the susceptibility rates of gatifloxacin and levofloxacin are $95-100 \%$ and the rates of trimethoprim/ sulfamethoxazole and piperacillin/tazobactam are $79 \%$ and $71 \%$, respectively.[3] However, our antimicrobial susceptibility test results only showed a $67 \%$ susceptibility rate for levofloxacin, $30 \%$ for trimethoprim/sulfamethoxazole, and $20 \%$ for piperacillin/tazobactam. This difference is assumed to be the result of widespread empirical use of anti-pseudomonal antibiotics for nosocomial infections and subsequent selection of a resistant strain in our hospital environment. 
However, the possibility of different antimicrobial susceptibility patterns for this organism in Korea should also be considered.

Even if effective antibiotic use after culture results were available was an independent predictor of 14-day mortality in a Taiwanese study (odds ratio, 0.31; $\mathrm{p}=0.049$ ),[7] effective antibiotics were only provided for $12(40 \%)$ patients in this study. Because E. meningoseptica is a strange organism to clinicians, most might think its presence in culture specimens is due to contamination or colonization. They would rather use carbapenems and vancomycin to treat bacterial pneumonia rather than use an antibiotic effective against E. meningoseptica based on culture results. However, appropriate antibiotics trended to be more commonly used in survivors, despite a lack of statistical significance $(42.9 \%$ vs. $33.3 \%, p=0.704$ ) in our study. Considering the high rate of E. meningoseptica-related mortality, if this organism was isolated from specimens of patients with an increased risk of infection, clinicians should pay attention to the disease progression and evaluate the response to current antibiotics, and if possible, consider using effective antibiotics based on culture reports.

This study had several limitations inherent to its retrospective nature. First, the bacterial culture and antimicrobial susceptibility results were obtained by using only MIC standards $(\mu \mathrm{g} / \mathrm{mL})$ of Gram-negative bacilli with an automatic system, and thus, antimicrobial susceptibility results for vancomycin and rifampin were not evaluated in this study. Because all specimens had been discarded, no further evaluations were performed. Second, our definitions to define E. meningoseptica infection were a little arbitrary, such as pneumonia. Third, because our sample size was too small to evaluate treatment outcomes and predictors of poor outcomes, whether E. meningoseptica infection truly affected the mortality rate or not was not clearly defined. Fourth, the data were obtained retrospectively from patients admitted to a single tertiary hospital; thus, the result should not be generalized.

Despite these limitations, considering the increasing number of reports on the clinical importance and high mortality rate caused by $E$. meningoseptica infection in adult patients with nosocomial infections, further studies are needed to establish its clinical impact and determine the most effective therapeutic strategy on a large scale in Korea.
ORCID

Doh Hyung Kim http://orcid.org/0000-0002-3567-4254

\section{References}

1) Bloch KC, Nadarajah R, Jacobs R: Chryseobacterium meningosepticum: an emerging pathogen among immunocompromised adults. Report of 6 cases and literature review. Medicine (Baltimore) 1997; 76: 30-41.

2) Weaver KN, Jones RC, Albright R, Thomas Y, Zambrano $\mathrm{CH}$, Costello $\mathrm{M}$, et al: Acute emergence of Elizabethkingia meningoseptica infection among mechanically ventilated patients in a long-term acute care facility. Infect Control Hosp Epidemiol 2010; 31: 54-8.

3) Kirby JT, Sader HS, Walsh TR, Jones RN: Antimicrobial susceptibility and epidemiology of a worldwide collection of Chryseobacterium spp: report from the SENTRY Antimicrobial Surveillance Program (19972001). J Clin Microbiol 2004; 42: 445-8.

4) Ceyhan M, Yildirim I, Tekeli A, Yurdakok M, Us E, Altun B, et al: A Chryseobacterium meningosepticum outbreak observed in 3 clusters involving both neonatal and non-neonatal pediatric patients. Am J Infect Control 2008; 36: 453-7.

5) Güngör S, Ozen M, Akinci A, Durmaz R: A Chryseobacterium meningosepticum outbreak in a neonatal ward. Infect Control Hosp Epidemiol 2003; 24: 613-7.

6) Chiu CH, Waddingdon M, Greenberg D, Schreckenberger PC, Carnahan AM: Atypical Chryseobacterium meningosepticum and meningitis and sepsis in newborns and the immunocompromised, Taiwan. Emerg Infect Dis 2000; 6: 481-6.

7) Hsu MS, Liao CH, Huang YT, Liu CY, Yang CJ, Kao KL, et al: Clinical features, antimicrobial susceptibilities, and outcomes of Elizabethkingia meningoseptica (Chryseobacterium meningosepticum) bacteremia at a medical center in Taiwan, 1999-2006. Eur J Clin Microbiol Infect Dis 2011; 30: 1271-8.

8) Hung PP, Lin YH, Lin CF, Liu MF, Shi ZY: Chryseobacterium meningosepticum infection: antibiotic susceptibility and risk factors for mortality. J Microbiol Immunol Infect 2008; 41: 137-44. 
9) Pokrywka M, Viazanko K, Medvick J, Knabe S, McCool S, Pasculle AW, et al: A Flavobacterium meningosepticum outbreak among intensive care patients. Am J Infect Control 1993; 21: 139-45.

10) Fujita J, Hata Y, Irino S: Respiratory infection caused by Flavobacterium meningosepticum. Lancet 1990; 335: 544.

11) Brown RB, Phillips D, Barker MJ, Pieczarka R, Sands M, Teres D: Outbreak of nosocomial Flavobacterium meningosepticum respiratory infections associated with use of aerosolized polymyxin B. Am J Infect Control 1989; 17: 121-5.

12) Jean SS, Lee WS, Chen FL, Ou TY, Hsueh PR: Elizabethkingia meningoseptica: an important emerging pathogen causing healthcare-associated infections. J Hosp Infect 2014; 86: 244-9.

13) Pereira GH, Garcia Dde O, Abboud CS, Barbosa VL, Silva PS: Nosocomial infections caused by Elizabethkingia meningoseptica: an emergent pathogen. Braz J Infect Dis 2013;17: 606-9.

14) Koo HM, Doh FM, Kim EJ, Kang EW, Shin SK, Chang TI: A case of peritonitis caused by Chryseobacterium meningosepticum in a patient treated with peritoneal dialysis. Korean J Nephrol 2011; 30: 339-42.

15) Kim SJ, Lee SM, Park SY, Ryu SH, Park YB, Eom JS, et al: A case of community-acquired pneumonia and sepsis caused by Chryseobacterium meningosepticum. Korean J Med 2010; 79: 453-6.

16) Yoon HS: Two cases of Chryseobacterium meningosepticum infection in a neonatal intensive care unit. Korean J Pediatr 2007; 50: 698-701.

17) Lee YS, Lee YH, Kim SN, Kang DH, Choi KB, Yoon KI, et al: A case of Chryseobacterium (Flavobacterium) meningosepticum peritonitis in a patient on CAPD. Korean J Nephrol 2002; 21: 815-9.

18) Choi HY, Cho YK, Yoo SJ, Kim KS: Ventriculitis by nosocomial Flavobacterium meningosepticum in neonates: neurosonographic findings. J Korean Soc Med Ultrasound 1992; 11: 95-100.

19) Yang YS, Chun JW, Koh JW: Keratitis with Elizabethkingia meningoseptica occurring after contact lens wear: a case report. Korean J Ophthalmol 2013; 27: 133-6.

20) Kim J, Park YJ, Shin S, Kim Y, Park SH: Three adult cases of Elizabethkingia meningoseptica infection in a Korean hospital. Korean J Clin Microbiol 2011; 14: 153-7.

21) Yum JH, Lee EY, Hur SH, Jeong SH, Lee H, Yong D, et al: Genetic diversity of chromosomal metallo-betalactamase genes in clinical isolates of Elizabethkingia meningoseptica from Korea. J Microbiol 2010; 48: 358-64.

22) Bellais S, Poirel L, Leotard S, Naas T, Nordmann P: Genetic diversity of carbapenem-hydrolyzing metallobeta-lactamases from Chryseobacterium (Flavobacterium) indologenes. Antimicrob Agents Chemother 2000; 44: 3028-34.

23) González LJ, Vila AJ: Carbapenem resistance in Elizabethkingia meningoseptica is mediated by metallo- $\beta$ lactamase BlaB. Antimicrob Agents Chemother 2012; 56: $1686-92$

24) Lee NY, Kim MS, Kim MN, Kim MJ, Kim S, Kim SI, et al: Annual report on external quality assessment in clinical microbiology laboratory in Korea (2008). J Lab Med Qual Assur 2009; 31: 13-27.

25) Lin YT, Chiu CH, Chan YJ, Lin ML, Yu KW, Wang FD, et al: Clinical and microbiological analysis of Elizabethkingia meningoseptica bacteremia in adult patients in Taiwan. Scand J Infect Dis 2009; 41: 628-34.

26) Fraser SL, Jorgensen JH: Reappraisal of the antimicrobial susceptibilities of Chryseobacterium and Flavobacterium species and methods for reliable susceptibility testing. Antimicrob Agents Chemother 1997; 41: 2738-41.

27) Amer MZ, Bandey M, Bukhari A, Nemenqani D: Neonatal meningitis caused by Elizabethkingia meningoseptica in Saudi Arabia. J Infect Dev Ctries 2011; 5: 745-7.

28) Lee SW, Tsai CA, Lee BJ: Chryseobacterium meningosepticum sepsis complicated with retroperitoneal hematoma and pleural effusion in a diabetic patient. J Chin Med Assoc 2008; 71: 473-6.

29) Tizer KB, Cervia JS, Dunn AM, Stavola JJ, Noel GJ: Successful combination vancomycin and rifampin therapy in a newborn with community-acquired Flavobacterium meningosepticum neonatal meningitis. Pediatr Infect Dis J 1995; 14: 916-7.

30) Tekerekoglu MS, Durmaz R, Ayan M, Cizmeci Z, Akinci A: Analysis of an outbreak due to Chryseobacterium meningosepticum in a neonatal intensive care unit. New Microbiol 2003; 26: 57-63. 